\title{
Top-Seeded Solution Crystal Growth, Morphology, Optical and Thermal Properties of $\mathrm{Ba}_{3}\left(\mathrm{ZnB}_{5} \mathrm{O}_{10}\right) \mathrm{PO}_{4}$
}

Hongwei Yu, ${ }^{\dagger}$ Jacqueline Cantwell, ${ }^{\dagger}$ Hongping Wu, ${ }^{\dagger}$ Weiguo Zhang, ${ }^{\dagger}$ Kenneth R. Poeppelmeier, ${ }^{*}$ and P. Shiv Halasyamani ${ }^{*} \dagger$

${ }^{\dagger}$ Department of Chemistry, University of Houston, 112 Fleming Building, Houston, Texas 77204-5003, United States

${ }^{\ddagger}$ Department of Chemistry, Northwestern University, 2145 Sheridan Road, Evanston, IL 60208-3133, United States

E-mail:psh@uh.edu (P.S. Halasyamani) 
Figure S1. The theoretical morphology of BZBP.

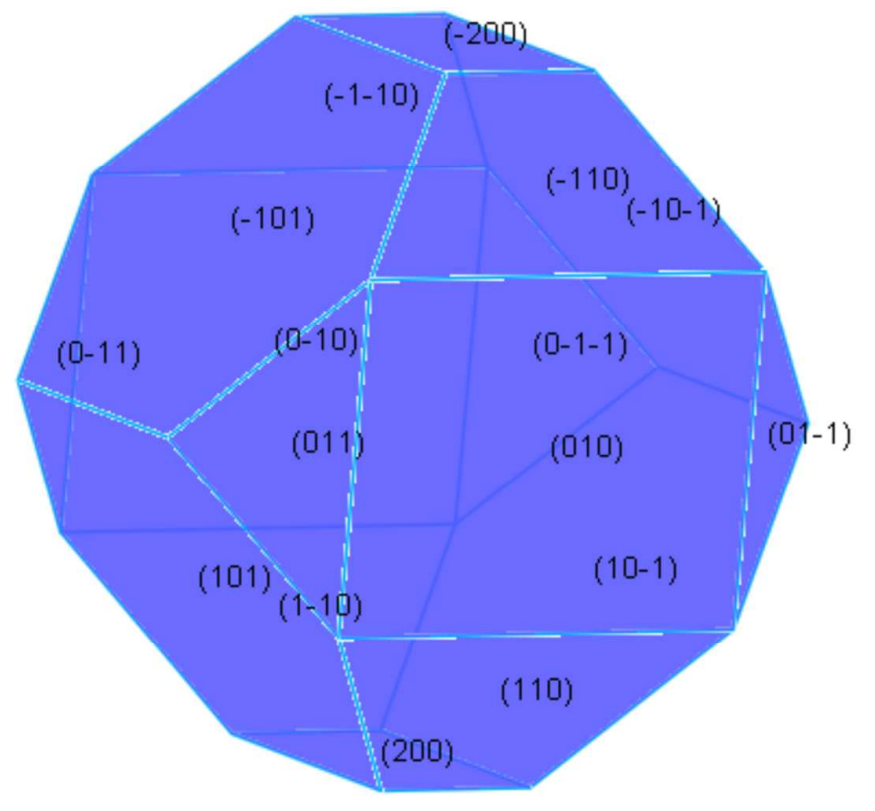


Figure S2. The theoretical morphologies of BZBP viewed along (a) [001]-, (b) [010]and (c) [101]-directions.

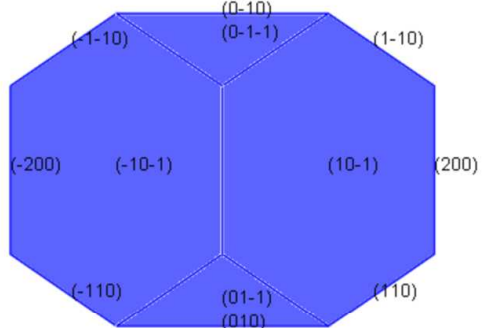

(a)

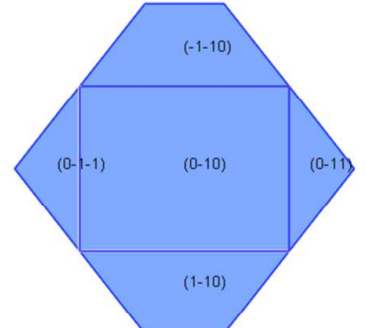

(b)

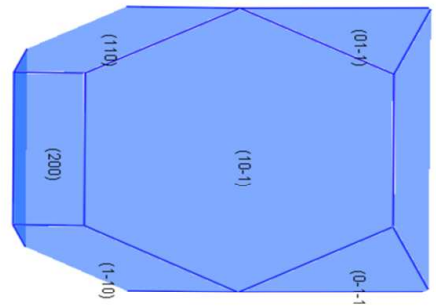

(c) 
Table S1 The comparison of the absorption edges and the limits of type-I phase matching (PM) SHG wavelength in BZBP, borate NLO crystals and borophosphate NLO crystals.

\begin{tabular}{lll}
\hline Compounds & $\begin{array}{l}\text { Absorption edges } \\
(\mathrm{nm})\end{array}$ & $\begin{array}{l}\text { The limit of type-I } \\
\text { PM SHG wavelength }(\mathrm{nm})\end{array}$ \\
\hline $\mathrm{KBe}_{2} \mathrm{BO}_{3} \mathrm{~F}_{2}(\mathrm{KBBF})$ & $147^{\mathrm{s} 1}$ & $164^{\mathrm{s} 1}$ \\
$\beta-\mathrm{BaB}_{2} \mathrm{O}_{4}(\beta-\mathrm{BBO})$ & $185^{\mathrm{s} 2}$ & $205^{\mathrm{s} 2}$ \\
$\mathrm{CsLiB}_{6} \mathrm{O}_{10}(\mathrm{CLBO})^{\mathrm{s} 3}$ & $180^{\mathrm{s3}}$ & $238^{\mathrm{s} 3}$ \\
$\mathrm{LiB}_{3} \mathrm{O}_{5}(\mathrm{LBO})^{\mathrm{s} 2}$ & $155^{\mathrm{s} 2}$ & $277^{\mathrm{s} 2}$ \\
$\mathrm{Ba}_{3}\left(\mathrm{ZnB}_{5} \mathrm{O}_{10}\right) \mathrm{PO}_{4}(\mathrm{BZBP})$ & 180 & 365 \\
$\mathrm{BPO}_{4}$ & $130^{\mathrm{s} 4}$ & Cannot be direct angle phase \\
$\beta-\mathrm{Zn}_{3} \mathrm{BPO}_{7}$ & & matching ${ }^{\mathrm{s} 4}$ \\
$\mathrm{SrBPO}_{5}$ & $250^{\mathrm{s} 5}$ & $399^{\mathrm{s5}}$ \\
\hline
\end{tabular}




\section{References}

(S1) Chen, C. T.; Wang, G. L.; Wang, X. Y.; Xu, Z. Y. Appl. Phys. B 2009, 97, 9.

(S2) Chen, C.; Sasaki, T.; Li, R. K.; Wu, Y.; Lin, Z.; Mori, Y.; Hu, Z.; Wang, J.; Uda, S.;

Yoshimura, M.; Kaneda, Y., Nonlinear Optical Borate Crystals. 2012

(S3) Mori, Y.; Kuroda, I.; Nakajima, S.; Sasaki, T.; Nakai, S. Appl. Phys. Lett. 1995, 67, 1818.

(S4) Li, Z. H.; Lin, Z. H.; Wu, Y. C.; Fu, P. Z.; Wang, Z. Z.; Chen, C. T. Chem. Mater. 2004, 16, 2906.

(S5) Wang, G.; Wu, Y.; Fu, P.; Liang, X.; Xu, Z.; Chen, C. Chem. Mater. 2002, 14, 2044.

(S6) Pan, S. L.; Wu, Y. C.; Fu, P. Z.; Zhang, G. C.; Li, Z. H.; Du, C. X.; Chen, C. T. Chem. Mater. 2003, 15, 2218. 